\title{
Evaluación de Tecnologías Sanitarias (ETESA) una Visión Global del Concepto y de sus Alcances
}

\author{
Health Technology Assessment (HTA). An Overview of the Concept and its Scope
}

Carlos Manterola1; Tamara Otzen ${ }^{1,2}$; Mayra Castro $^{3,4}$ \& Luis Grande L $^{5}$

MANTEROLA, M.; OTZEN, T.; CASTRO, M. \& GRANDE, L. Evaluación de tecnologías sanitarias (ETESA). Una visión global del concepto y de sus alcances. Int. J. Morphol., 36(3):1134-1142, 2018.

RESUMEN: La progresivas preocupación de los países para optimizar el acceso, la eficiencia y la calidad de la salud, han impulsado la utilización más apropiada de las intervenciones en salud. Por ende, el interés tanto de profesionales sanitarios como de tomadores de decisiones en salud, ha sido orientado hacia la medicina basada en la evidencia, la eficacia comparativa y la Evaluación de Tecnologías Sanitarias (ETESA). Aunque los conceptos anteriormente señalados son convergentes en sus características, no son sinónimos. Sin embargo, todos estos, se basan en la orientación sistemática de pruebas y el enfoque en resultados relevantes para el paciente entre otras. Como consecuencia de todo esto, el interés no sólo implica los conceptos de eficacia, efectividad y eficiencia; sino que también en práctica clínica, costes y transparencia. En este artículo se resumen los conceptos de economía de la salud, evaluación económica, tecnología sanitaria (TS) y ETESA. Luego, se comenta el ciclo de vida de una TS, razones para implementar un programa de ETESA; para finalizar con algunos ejemplos de TS emergentes, comentarios respecto de la evidencia científica en la ETESA; y algunos ejemplos de estudios de ETESA en la práctica clínica cotidiana.

PALABRAS CLAVE: Evaluación de Tecnologías Sanitarias; Economía de la salud; Tecnologías sanitarias; Medicina basada en evidencia.

Económia de la salud: En un clima de creciente demanda de nuevos y mejores servicios de atención sanitaria y de restricciones económicas significativas, las disciplinas de economía de la salud y de evaluación de tecnologías sanitarias (ETESA), se han convertido en marcos referenciales de la práctica clínica basada en la evidencia (Goeree \& Diaby, 2013).

Economía de la salud, es una ciencia económica que estudia la acción de las leyes, objetivos económicos, condiciones, factores que aseguran la satisfacción máxima de las necesidades de la sociedad en la atención médica y protección de la salud de la población, de la asignación eficiente de la salud y de los recursos de atención con gastos mínimos de trabajo social y manual (Gray \& Lorgelly, 2010; Goeree \& Diaby). Integra las teorías económicas, sociales, clínicas y epidemiológicas, para el estudio de los mecanismos determinantes y condicionantes de la producción, distribución, consumo y financiamiento de los servicios de salud (Oppong et al., 2013).
Evaluación económica: La evaluación económica, es un conjunto de técnicas que se utilizan para comparar las opciones abiertas para el decisor, en una situación de elección relacionada con un conjunto de posibles cursos de acción. Involucra una serie de aspectos objetivos, subjetivos y elementos éticos, sociales y políticos.

Tradicionalmente, la economía de la salud y evaluación económica han sido ampliamente utilizados en el desarrollo de políticas y toma de decisiones; y han ido teniendo un rol relevante en la información de decisiones clínicas individuales (Goeree \& Diaby).

Si se quiere maximizar el bienestar social, hay que tener en cuenta los costes de toda decisión que afectan directa o indirectamente la asignación de recursos; ya que la limitación de éstos y la búsqueda de mayor eficiencia, justifican plenamente la utilización de métodos de evaluación económica en la toma de decisiones (OPS, 2017; Tanvejsilp \& Ngorsuraches, 2014).

\footnotetext{
${ }^{1}$ Centro de Excelencia en Estudios Morfológicos y Quirúrgicos (CEMyQ), Universidad de La Frontera, Temuco, Chile.

${ }^{2}$ Universidad de Tarapacá, Arica, Chile.

${ }^{3}$ Instituto de Salud Materno Infantil (ISAMI), Ecuador.

${ }^{4}$ Programa de Doctorado en Ciencias Médicas, Universidad de La Frontera, Temuco, Chile.

${ }^{5}$ Institut Hospital del Mar d'Investigacions Mèdiques (IMIM), Barcelona, España.
} 
Tecnología sanitaria (TS): Las TS son equipos, dispositivos, instrumentos, técnicas, procedimientos médicos y quirúrgicos, medicamentos, programas sanitarios, sistemas de información, organización y de apoyo de los servicios de salud, destinados a la prevención, tratamiento y rehabilitación de situaciones clínicas específicas, y a mejorar la calidad de vida de los personas y de la sociedad (Tanvejsilp \& Ngorsuraches; Vallejos et al., 2014; OPS, 2017).

Evaluación de tecnologías sanitarias (ETESA). ETESA es un campo amplio con base en diferentes disciplinas; y en especial, de herramientas de economía en salud y evaluación económica (Goeree \& Diaby). Es muy útil para ayudar en el desarrollo de políticas sanitarias basadas en la mejor evidencia; y para orientar de mejor forma la asignación de los siempre escasos recursos en salud (Ju \& Hewson, 2014).

Por otro lado, ETESA es la forma integral de investigar las consecuencias técnicas, legales, económicas, éticas y sociales que ocurren a lo largo del tiempo, así como los efectos directos o indirectos, deseados e indeseados, que se derivan del uso de una TS (Kuhn-Barrientos, 2014; Gagnon, 2014; Lysdahl et al., 2016; OPS, 2017).

El objetivo de la ETESA, es contribuir a la toma de decisiones en la práctica clínica, en la implementación de políticas sanitarias; que incluye: planificación de gastos y recursos, con óptima asignación de los mismos, ya sean tecnológicos o de innovación y desarrollo (Youngkong, 2014).
El análisis estructurado de una ETESA requiere de: una TS, un conjunto de TS relacionadas, y de un problema relacionado con la TS. Los componentes del proceso de ETESA son la valoración de la TS y el informe del proceso (Teutsch \& Berger, 2005) (Fig. 1); posterior al cual se debe llegar a una recomendación respecto de la ETESA realizada (Fig. 2). Un ejemplo del desarrollo y alcance del espectro de las TS en América Latina y el Caribe se puede apreciar en la Figura 3 (OPS, 2000; Yglesias Bedoya, 2007).

Por otra parte, los criterios a considerar en ETESA son: eficacia, efectividad, eficiencia, utilidad; seguridad/riesgo; necesidad (demográfica, económica y epidemiológica); equidad, idoneidad e impacto social (ético, psicológico, organizativo, legal), etc. (Conde Olasagasti, 1998; Gagnon; Gagnon et al., 2014).

Eficacia: Expresa, el efecto o el resultado de una TS aplicada a individuos de una población determinada, para resolver un problema concreto, en condiciones ideales o experimentales (ensayos clínicos controlados, con asignación aleatoria). Sus limitaciones se relacionan con que: la generalización de los resultados es cuestionable, ya que éstos se evalúan en condiciones óptimas o de laboratorio; sólo se comparan efectos comunes de las alternativas (cuando no son comunes, se debe describir la probabilidad y magnitud de estos efectos). Se mide en función de actividades programadas y efectivamente realizadas (Actividades efectivamente realizadas / Actividades programadas).

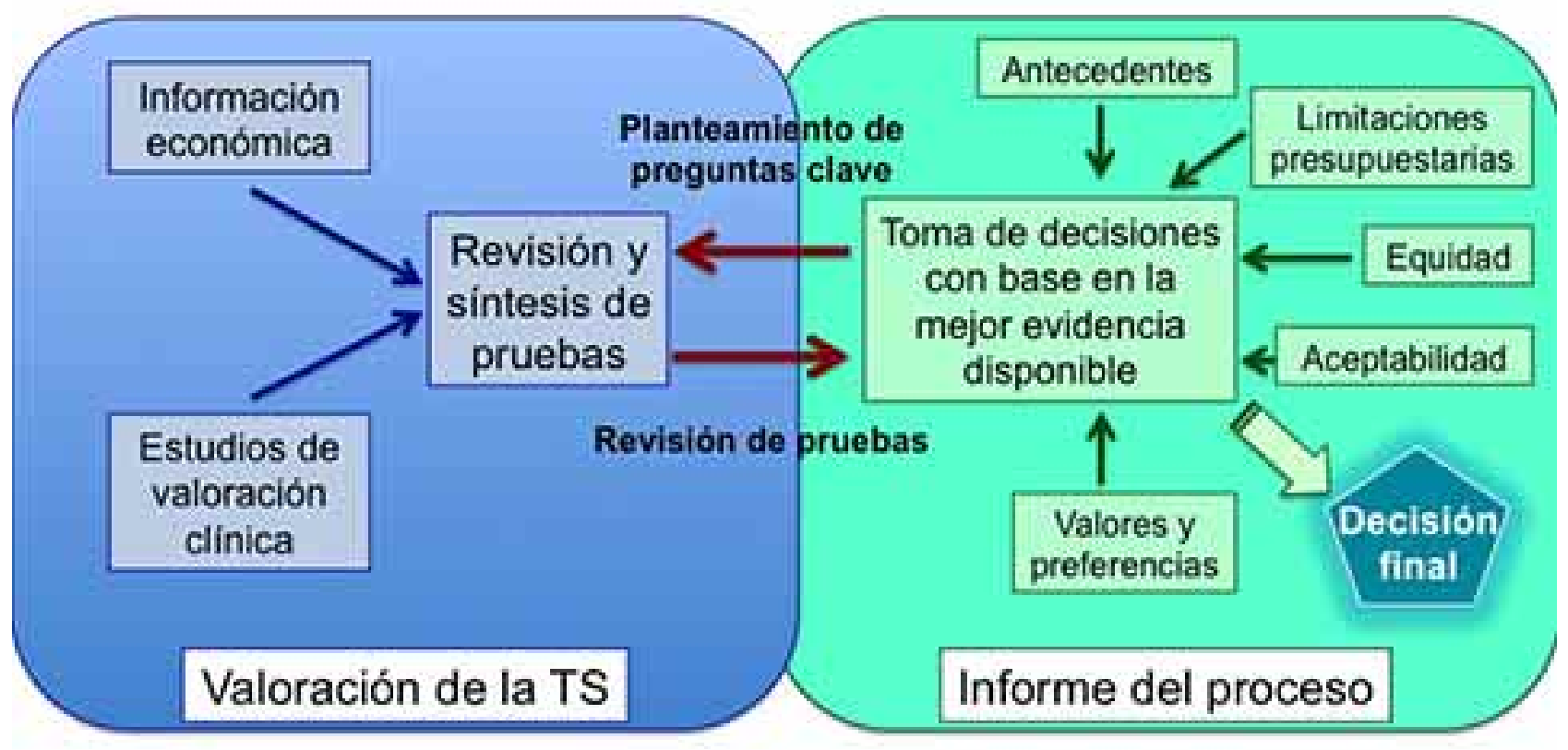

Fig. 1. Dos componentes elementales del proceso de ETESA. La valoración de la TS y el informe. Adaptado de "Evidence synthesis and evidence-based decision making; Related but distinct processes" (Teutsch \& Berger, 2005). 


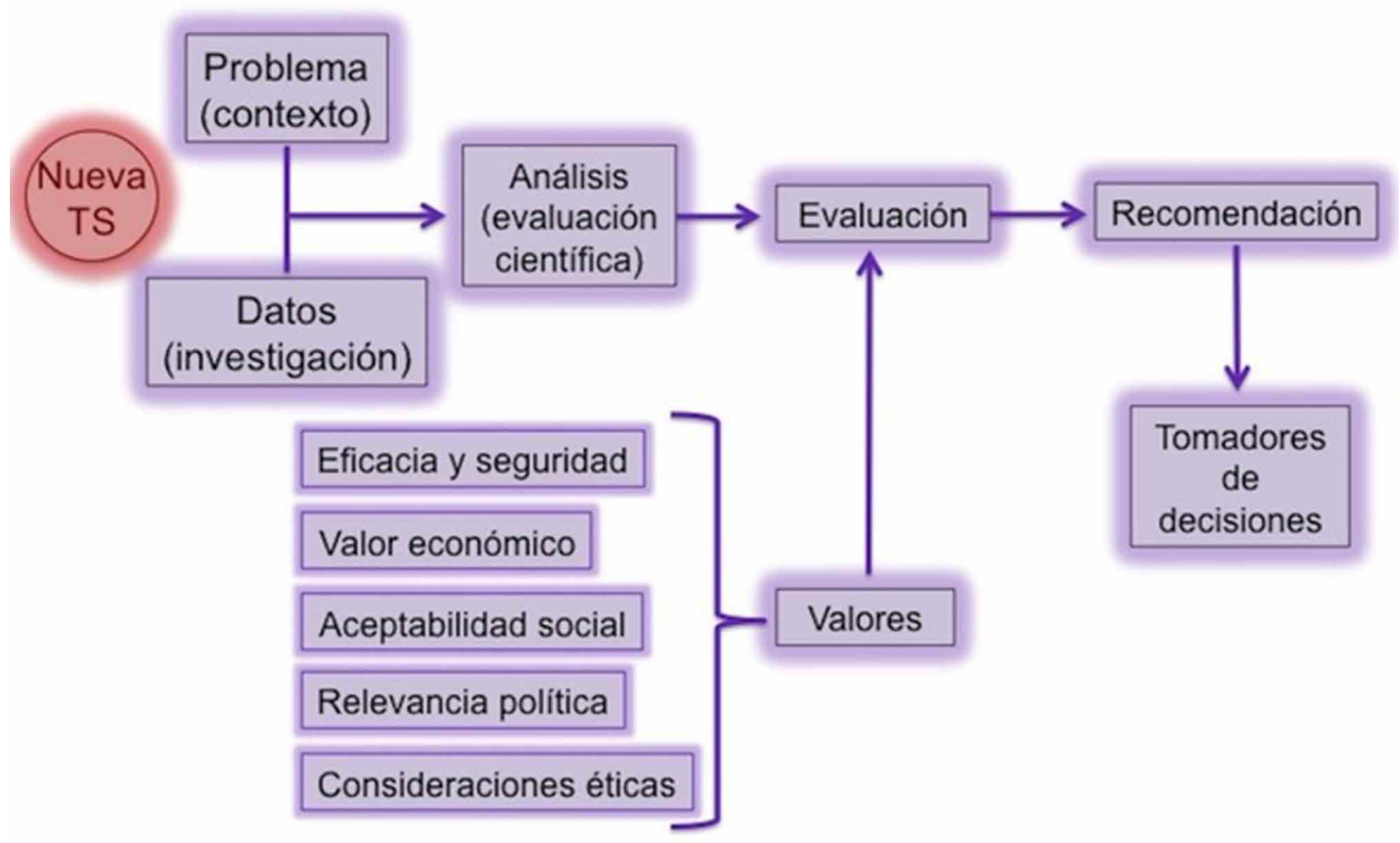

Fig. 2. Llegando a una recomendación de ETESA.

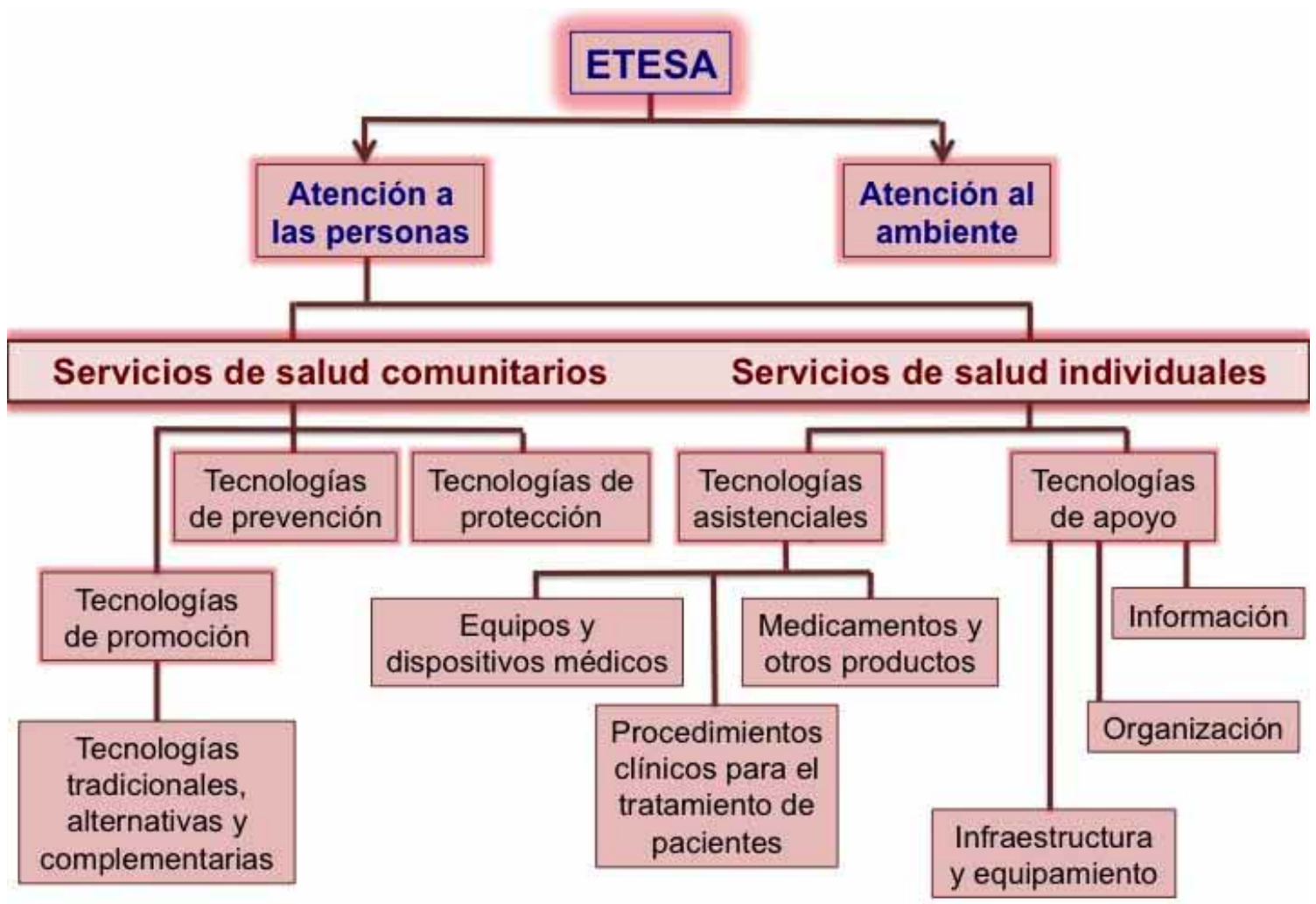

Fig. 3. El alcance del espectro de las Tecnologías de Atención a la Salud en América Latina y el Caribe. Adaptado de "El desarrollo de la evaluación de las tecnologías en salud en América Latina y el Caribe" (OPS, 2000; Yglesias Bedoya, 2007). 
Efectividad: Corresponde a la medida del efecto de una TS aplicada a individuos de una población determinada, para resolver un problema concreto, en la práctica clínica habitual (condiciones reales). Sus limitaciones se relacionan con que: se deben describir, de forma similar a la eficacia, los efectos no comunes que resultan del uso de TS comparadas. Aunque la generalización de resultados es más factible; la efectividad de una TS puede diferir entre pacientes, centros o lugares, debido a la variabilidad inter e intrapersonal. Se mide en función de objetivos alcanzados y propuestos (Objetivos alcanzados / Objetivos propuestos).

Eficiencia: Corresponde a la medida de relación entre resultados obtenidos o consecuencias derivadas del uso de una TS y los recursos invertidos en ello. Se mide en función del grado de utilización real y efectiva de los recursos en relación con la utilización programada (Utilización efectiva / Utilización programada del recurso).

Utilidad: Corresponde a la medida de la calidad de vida y su duración. Se refiere al grado subjetivo de bienestar que las personas experimentan en diferentes estados. En la evaluación, la calidad de vida relacionada con la salud (CVRS), se utiliza el concepto "valor asignado a la duración de la vida, modificado por deterioros, percepciones, estados funcionales y oportunidades sociales; sobre los que influyen enfermedad, tratamiento y política sanitaria". Se mide de forma multidimensional, utilizando cuestionarios; los que pueden ser específicos para una entidad nosológica (para una enfermedad o un rango estrecho de enfermedades); o genéricos (pueden ser aplicados a un amplio abanico de problemas sanitarios). Ahora bien, es importante comprender que la elección del instrumento de medición dependerá de la condición clínica en la que se aplique la TS que va a evaluarse, la variable que interesa medir y el entorno cultural de la población en la que se va a aplicar. Entre las más utilizadas, destacan los cuestionarios: QALY (años ajustados por calidad), Euro- QOL (el propio individuo valora su estado de salud, por ende, es útil para asignación de recursos sanitarios); AVAD (años de vida ajustados a discapacidad), HYES (años equivalentes de salud, que permite incorporar las preferencias del sujeto), etc. (Manterola et al., 2013, 2014).

Otros criterios a evaluar son: Seguridad/Riesgo, que corresponde a una medida de riesgo de los efectos no deseados que pueden aparecer como consecuencia de la aplicación de una TS; esto significa, que se ha de determinar que los beneficios del uso de la TS sean superiores a los riesgos. Necesidad, que corresponde a una medida de la demanda percibida de utilizar una TS (real o potencial), especifica en un contexto sanitario, según criterios demográficos, económicos y epidemiológicos. Equidad, que se evalúa mediante la posición socio-económica, que corresponde al desglose de salud alcanzada por intervenciones en subgrupos de la población según causas: legítimas e ilegítimas, modificables y no modificables (Espinoza \& Cabieses, 2014). Impacto social, que puede evaluarse mediante determinantes sociales de la salud; que permiten entre otras, informar y mejorar la toma de decisiones en salud, aportando recomendaciones para optimizar los resultados, con especial énfasis en los grupos más vulnerables (Conde Olasagasti; Adam \& Permanyer-Miralda, 2013).

Por otro lado, las técnicas para realizar evaluaciones económicas, tienen en común que los recursos utilizados son comparados con los resultados, los que se pueden expresar como: Eficacia, Efectividad, Utilidad y Beneficio. Estos, pueden ser evaluados de forma parcial y total. La evaluación parcial se puede realizar en términos de: Descripción de consecuencias, de costes y consecuencias, de eficacia y efectividad; y de análisis de costes. Por su parte, la evaluación total se puede realizar en términos de: Minimización de costes, Coste-efectividad, Coste-utilidad y Coste-beneficio.

\section{Estudios parciales:}

Estudios de descripción de las consecuencias: No hay comparación de las alternativas, ni análisis de costes. Suele evaluarse un programa o un servicio.

Descripción de costes: Se analiza el comportamiento de costes, sin considerar las alternativas ni consecuencias; por ejemplo estudios de coste por enfermedad.

Descripción de costes y consecuencias: No se consideran alternativas. Se incluyen solamente los costes y resultados de una enfermedad.

Análisis de la eficacia y efectividad: Consideran alternativas y resultados, sin embargo adolecen de estimaciones de coste.

Análisis de costes: Comparan alternativas y tienen en cuenta costes, pero no incluyen los resultados.

\section{Estudios totales o completos:}

Minimización de costes: Es el más simple. Se comparan dos o más alternativas que tienen un mismo resultado sanitario (efectividad). Sólo se puede identificar la alternativa menos costosa.

Coste-efectividad: Útil para comparar dos o más alternativas, donde los recursos se expresan en unidades monetarias y los efectos sobre la salud en unidades específicas no monetarias de efectividad (medidas de efectividad). Permite 
expresar los efectos en las mismas unidades utilizadas en ensayos clínicos y en la práctica clínica. Sin embargo, sólo permite seleccionar entre opciones similares y que tengan efectos medidos en la misma unidad; por lo cual, los resultados pueden presentarse de dos formas: Coste-efectividad media $($ Coste $/$ Efectividad $=$ pesos por unidad de efectivi dad); Coste-efectividad incremental (Coste A - Coste B / Efecto A - Efecto B).

Coste-beneficio: Su característica principal es que tanto costos como efectos de las alternativas comparadas se miden en unidades monetarias; por ende, permite comparar opciones cuyos resultados se miden en unidades diferentes, lo que es útil para comparar alternativas de áreas distintas. De este modo, los resultados de un análisis de coste-beneficio, pueden expresarse como cocientes de coste-beneficio ó como valor neto (Coste / Beneficio ó Coste - Beneficio).

Coste-utilidad: Permite integrar en el análisis no sólo cantidad de vida ganada (años de vida), sino calidad de vida obtenida. Las unidades obtenidas son Años de Vida Ajustados por Calidad (AVAC). Permite comparar: diferentes tipos de intervenciones o programas sanitarios distintos. Contempla además cantidad y calidad de vida en los sujetos a los que se dirige. Los resultados se presentan de forma similar a los de los estudios de coste-efectividad, substituyendo el denominador de la fracción por los AVAC, es decir Coste / AVAC.

Ciclo de vida de una TS: Es el proceso de evolución que tiene una TS en el tiempo. En una primera etapa, sólo se encuentra a disposición de unos pocos consumidores, para posteriormente ser conocida por todo el mercado; y finalmente, cuando se encuentra saturada u obsoleta, saldrá del mercado.
Por ello, toda TS tiene un ciclo de vida, que se diferencia en la duración de cada fase. Para estudiar los ciclos de las TS se genera una "curva S" (Foster, 1986); con la que se puede determinar la duración de cada fase del ciclo de vida de una TS (Fig. 4); de modo tal que, a medida que aumenta su nivel de conocimiento requerirá de mayores esfuerzos para incrementar el rendimiento esperado de la TS (Banta et al.).

\section{Ejemplos de TS emergentes}

La evaluación económica, no es la panacea frente a los problemas de limitación de recursos. Sin embargo, permite reducir arbitrariedades en la toma de decisiones sobre el uso de los recursos disponibles. Por ejemplo:

1.Dispositivos de asistencia ventricular percutáneos (Health Quality Ontario, 2017).

2.Tecnologías para la reducción de patógenos aplicadas al plasma para uso clínico (Cicchetti et al., 2016).

3.Tratamiento de la infección por Clostridium difficile con microbiota fecal (Health Quality Ontario, 2016a).

4. Pruebas cutáneas para la rinitis alérgica (Health Quality Ontario, 2016b).

5.Uso clínico de los medios sociales para mejorar la calidad de la atención en salud mental (Di Napoli et al., 2015).

6. Diseños de punto final en estudios de dolor crónico (Rycroft et al., 2015).

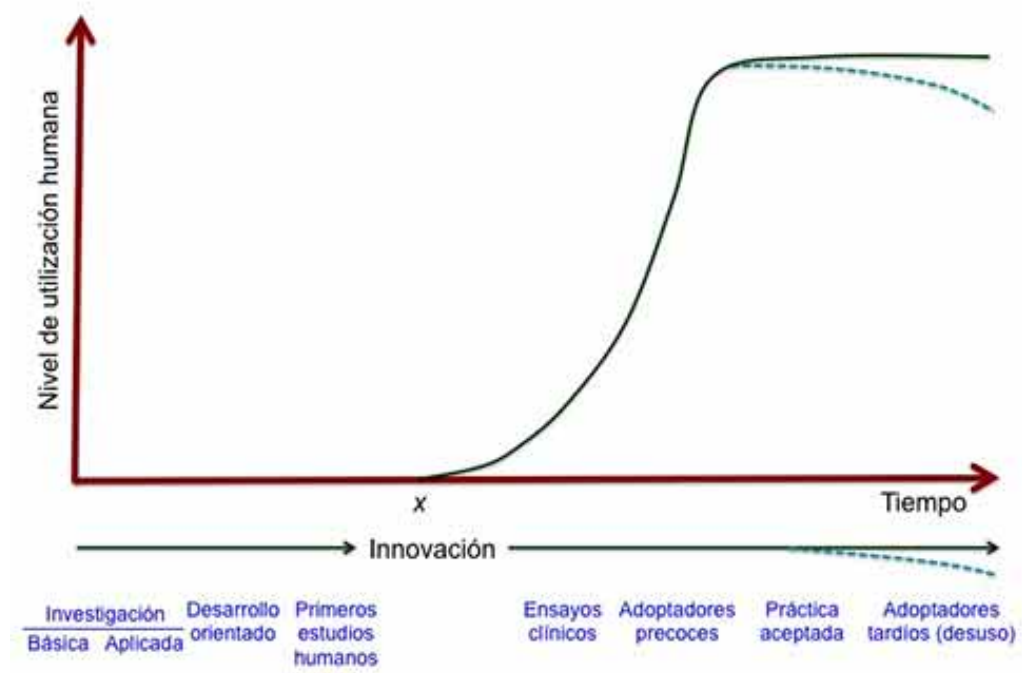

Fig. 4. El ciclo de vida de la tecnología. Adaptado de "Toward rational technology in medicine: considerations for health policy" (Banta et al., 1981). 


\section{Razones para implementar un programa de ETESA}

Existe una serie de razones para implementar un programa de ETESA; entre las que destacan: el incremento creciente del gasto en salud (Figs. 5 y 6), en especial en presencia de recursos limitados; la presión del sistema y de la industria de salud por introducir una TS sin valoración suficiente; los registros disponibles sobre el gasto de un país en TS respecto del gasto total en salud (en especial cuando no existen registros, o son insuficientes); la valoración inexacta de los usuarios respecto de calidad y seguridad en atención sanitaria, por desconocimiento del impacto social y ético. Porque aunque las nuevas TS pueden mejorar la calidad de la atención en salud, no siempre ofrecen ganancia neta en coste-efectividad, seguridad e impacto social, etc. (Castillo-Riquelme \& Santelices, 2014).

Entonces, las motivaciones para implementar un programa de ETESA, tienen que ver con proporcionar certidumbre y reducir riesgos en la toma de decisiones; conocer las alternativas técnicas, sus costes y beneficios; lograr un impacto en los resultados sanitarios; favorecer el uso racional de recursos con resultados efectivos; y generar información útil en la toma de decisiones en salud, en los diferentes niveles del sistema.
Por otra parte, existen una serie de barreras a la implementación de un programa de ETESA (CastilloRiquelme \& Santelices); como la falta datos e información para aplicar esta evaluación (implica en sus inicios importar datos y aplicarlos a la realidad en la que se requiere implementar); la resistencia de la industria farmacéutica, los equipos clínicos y los profesionales sanitarios; los intereses comerciales de implementación y masificación de TS que no aportan a la población; y, las dificultades en la implementación, porque existen pocas pruebas respecto a que las evaluaciones influyan en la toma de decisiones en el uso de TS o en los resultados sanitarios.

\section{Etesa y evidencia científica}

El análisis de la evidencia distingue entre estudios de buena y mala calidad. Es posible que se haya realizado investigación científica sobre un tema, pero que no se realizara con una metodología de calidad. En ese caso las conclusiones se basan en evidencia científica de mala calidad. La atención sanitaria basada en la evidencia científica es aquella que está fundamentada en la utilización de información válida y fiable, resultado de la investigación, evaluada rigurosamente y recogida en un documento o informe (Conde Olasagasti).

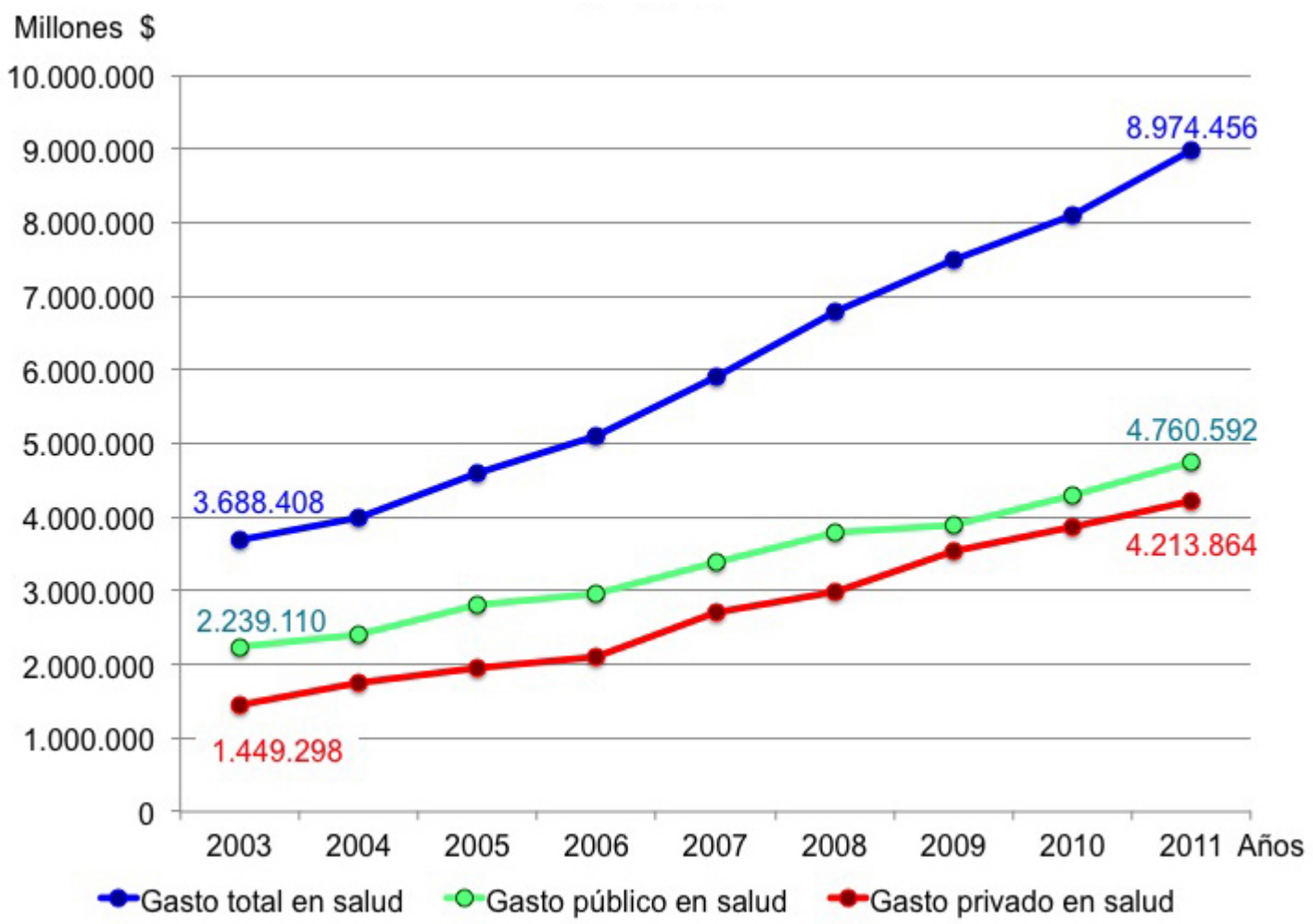

Fig. 5. Evolución del gasto en salud pública y privada de Chile entre 2003 y 2011. 


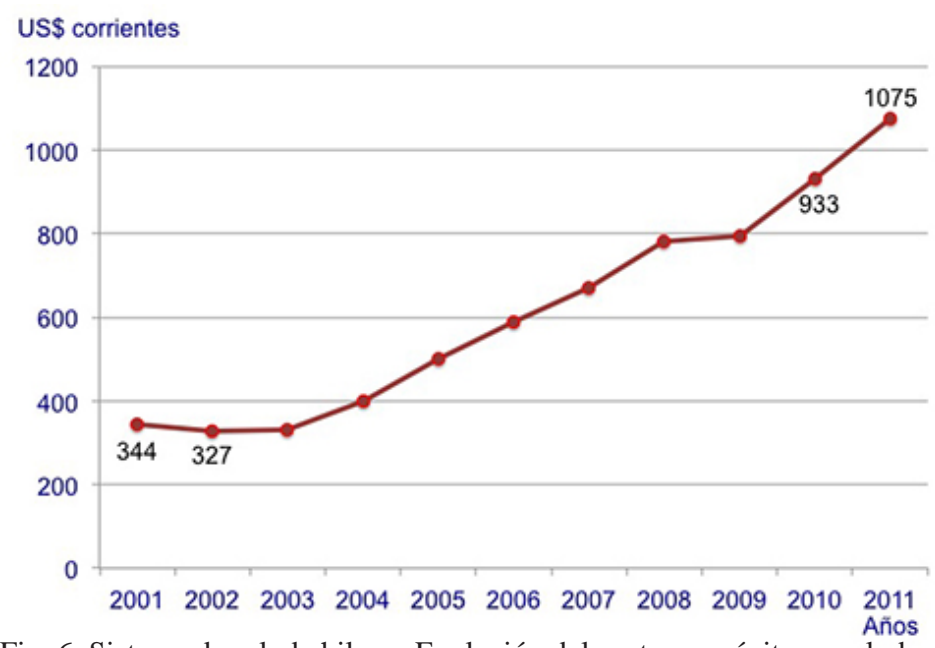

Fig. 6. Sistema de salud chileno. Evolución del gasto per cápita en salud 2001-2011.

¿Qué ocurre cuando no hay evidencias? Se pueden conducir estudios de investigación, esperar el resultado de estudios en marcha y generar consenso de expertos.

¿Se pueden implantar una TS sin evaluación previa? Ninguna tecnología se debería implantar sin una previa evaluación. Esto, bajo la premisa de garantizar la seguridad del paciente. Sin embargo, esto no siempre es así, puesto que, algunas TS se utilizan en la práctica con estudios de baja o muy baja calidad (sólo por recomendaciones de expertos).

\section{Ejemplo 1.}

¿Cuál es la efectividad de la colecistectomía laparoscópica (CL)? Se planteó como pregunta secundaria ¿Qué queremos saber de la CL? Luego, se estructuraron los siguientes apartados: CL vs. colecistectomía laparotómica, CL en grupos especiales de pacientes, Profilaxis antibiótica en $\mathrm{CL}$, Colangiografía intraoperatoria de rutina en CL, Lesiones de la vía biliar asociadas a la CL, Utilización de variantes técnológicas para la realización de una CL, y CL como cirugía mayor de corta estancia (Manterola et al., 2007a).

A continuación, se realizaron búsquedas sensibles en las siguientes bases de datos: Biblioteca Cochrane Plus 2006, MEDLINE (PubMed, 1986-2006), EMBASE (Dialog Star; 1986-2006), TRIP Database y Agencias Internacionales de Evaluación de Tecnologías; considerando todo tipo de estudios sobre efectividad de CL. Se realizó una clasificación global de la evidencia (aplicando la propuesta GRADE que clasifica la evidencia en alta, moderada y baja (Guyatt et al., 2001), teniendo en cuenta el diseño de los estudios (aplicando la propuesta de niveles de evidencia del Centro de Medicina Basada en la Evidencia de Oxford (CEBM, 2009); la validez interna (se usaron los criterios propuestos por el Scottish
Intercollegiate Guidelines Network (Scottish Intercollegiate Guidelines Network, 2004), de resumen y se aplicó escala de Jadad), la evaluación de si la evidencia era directa o indirecta, la consistencia y la precisión de los resultados, así como la existencia de otros factores, como el posible sesgo de publicación. Con esta metodología, se obtuvieron los siguientes resultados:

CL vs. colecistectomía laparotómica: Se encontró evidencia a partir de 3 revisiones sistemáticas (RS), 2 ensayos clínicos (EC), 1 guía de práctica clínica (GPC) y 2 estudios de cohortes. La evidencia recogida permitió resumir los siguientes hechos: $\mathrm{La}$ CL respecto a la técnica abierta, se asocia a un mayor tiempo operatorio, una menor estancia hospitalaria y un curso postoperatorio con una mejor calidad de vida en pacientes con colecistitis crónica y aguda. La CL se muestra como un procedimiento seguro aunque existe un riesgo de lesiones de la vía biliar asociadas con esta técnica. Estas lesiones se asocian a la curva de aprendizaje del procedimiento y generalmente requieren cirugías de reparación complejas que implican costes significativos (Calidad alta).

CL en grupos especiales de pacientes: Se encontró evidencia a partir de 1 RS, 3 EC, 7 estudios de cohortes, 1 estudio de casos y controles (CC) y 7 series de casos (SC). La evidencia recogida permitió resumir los siguientes hechos: $\mathrm{La}$ CL se muestra como un procedimiento seguro en algunos subgrupos especiales de pacientes como ancianos, embarazadas, pacientes con cirrosis e hipertensión portal Child A y B y EPOC (Calidad baja).

Profilaxis antibiótica en CL: Se encontró evidencia a partir de 1 RS, 1 EC y 1 GPC. La evidencia recogida permitió resumir los siguientes hechos: el uso en pacientes de bajo riesgo, respecto a no usarla, no ofrece beneficios adicionales en términos de disminución de la tasa de complicaciones infecciosas postoperatorias (Calidad alta).

Colangiografía intraoperatoria de rutina en CL: Se encontró evidencia a partir de 1 estudio de cohorte y $1 \mathrm{SC}$. La evidencia disponible sobre la necesidad de la realización de una colangiografía intraoperatoria de rutina en el curso de la CL es escasa (Calidad baja).

Lesiones de la vía biliar asociadas a la CL: Se encontró evidencia a partir de $1 \mathrm{CC}, 2$ estudios de corte transversal y 5 SC. La evidencia recogida permitió resumir los siguientes hechos: Existe riesgo de lesiones de la vía biliar asociadas. Se asocian a la curva de aprendizaje. Generalmente requieren cirugías de reparación complejas que implican costes significativos (Calidad moderada). 
Utilización de variantes tecnológicas: Se encontró evidencia a partir de $11 \mathrm{EC}, 1$ estudio de cohortes y $5 \mathrm{SC}$. La evidencia recogida permitió resumir los siguientes hechos: $\mathrm{Se}$ han descrito multiplicidad de variantes para la realización de una CL. Entre estas se encuentran la CL sin neumoperitoneo, la reducción del número de puertos de acceso, la utilización de "mini instrumentos", el uso de sistemas robóticos y la utilización de diversos sistemas de disección. No obstante la evidencia disponible no permite conocer la eficacia relativa de las mismas (Calidad moderada).

CL como cirugía mayor de corta estancia: Se encontró evidencia a partir de 1 estudio de cohortes y 4 SC. No hay evidencia de mayores complicaciones en CL en régimen de cirugía mayor ambulatoria o de corta estancia (Calidad baja).

\section{Ejemplo 2.}

¿Cuál es la efectividad de las resecciones hepáticas por vía laparoscópica? Se planteó como pregunta secundaria ¿Qué queremos saber de las resecciones hepáticas por vía laparoscópica? Luego, se estructuraron los siguientes apartados: Tratamiento laparoscópico de los quistes hepáticos, de los tumores hepáticos, y resecciones hepáticas vía laparoscópica (Manterola et al., 2007b).

A continuación, se realizaron búsquedas sensibles en las siguientes bases de datos: Biblioteca Cochrane Plus 2006, MEDLINE (PubMed, 1986-2006), EMBASE (Dialog Star; 1986-2006), TRIP Database y Agencias Internacionales de Evaluación de Tecnologías; considerando todo tipo de estudios sobre efectividad de resecciones hepáticas vía laparoscópica. Se realizó una clasificación global de la evidencia (aplicando la propuesta GRADE que clasifica la evidencia en alta, moderada y baja (Guyatt et al.), teniendo en cuenta el diseño de los estudios (aplicando la propuesta de niveles de evidencia del Centro de Medicina Basada en la Evidencia de Oxford (CEBM); la validez interna (se usaron los criterios propuestos por el Scottish Intercollegiate Guidelines Network (Scottish Intercollegiate Guidelines Network), de resumen y se aplicó escala de Jadad), la evaluación de si la evidencia era directa o indirecta, la consistencia y la precisión de los resultados, así como la existencia de otros factores, como el posible sesgo de publicación. Con esta metodología, se obtuvieron los siguientes resultados:

Tratamiento laparoscópico de los quistes hepáticos: Se encontró evidencia a partir de $1 \mathrm{RS}, 1$ estudios de cohortes y 9 SC. La evidencia recogida permitió resumir los siguientes hechos: Existen diferentes opciones técnicas, que van desde el simple destechamiento de las lesiones a la resección total de éstas; sin embargo, la calidad de la evidencia sobre la eficacia del tratamiento laparoscópico de quistes hepáticos, respecto a la vía laparotómica, es baja. El riesgo de confundir un quiste hidatídico hepático con un quiste no parasitario del hígado, debe ser considerado antes de la toma de decisiones, para reducir riesgos de complicaciones intra y postoperatorias (Calidad baja).

Tratamiento laparoscópico de los tumores hepáticos: Se encontró evidencia a partir de 3 estudios de cohortes y 2 SC. La evidencia recogida permitió resumir los siguientes hechos: Existen diferentes opciones de tratamiento laparoscópico para los tumores hepáticos, entre las que destacan el tratamiento resectivo y las terapias no resectivas como la inyección de etanol, la ablación por crioterapia y radiofrecuencia, y la coagulación por microondas. Estas opciones terapéuticas podrían ser consideradas en pacientes con contraindicación quirúrgica formal (Calidad baja). No se dispone de estudios comparativos respecto a la eficacia y seguridad del tratamiento laparoscópico resectivo no resectivo en tumores hepáticos.

Resecciones hepáticas vía laparoscópica: Se encontró evidencia a partir de $1 \mathrm{RS}, 3$ estudios de cohortes y $8 \mathrm{SC}$. La evidencia recogida permitió resumir los siguientes hechos: Existe evidencia que las resecciones hepáticas por vía laparoscópica se asocian con menor estancia hospitalaria y una reducción de las pérdidas hemáticas para respecto de las resecciones por vía abierta. La evidencia disponible sobre la morbilidad y mortalidad de las resecciones hepáticas laparoscópicas es escasa. La información disponible sugiere que podría ser similar a la observada en las resecciones por vía abierta (Calidad baja). No se dispone de estudios comparativos sobre la eficacia y seguridad de las resecciones hepáticas por vía laparoscópica.

MANTEROLA, M.; OTZEN, T.; CASTRO, M. \& GRANDE, L. Health technology assessment (HTA). An overview of the concept and its scope. Int. J. Morphol., 36(3):1134-1142, 2018.

SUMMARY: The progressive concern of countries to optimize access, efficiency and quality of health have led to the most appropriate use of health interventions. Therefore, the interest of both health professionals and health decision makers has been oriented towards evidence-based policy, comparative efficacy and Health Technologies Assessment (HTA). Although the aforementioned concepts are converging in their characteristics, they are not synonymous. However, all these are based on systematic testing orientation and focus on relevant patient outcomes among others. As a consequence of all this, interest does not only imply the concepts of effectiveness, effectiveness and efficiency, but also in clinical practice, costs and transparency. This manuscript summarizes concepts of health economics, economic evaluation, health technology and HTA. Then, the life cycle of a health technology and the reasons for implementing an HTA program are discussed. Concluding with some 
examples of emerging health technologies, comments on the scientific evidence in HTA, and some examples of HTA studies in daily clinical practice.

KEY WORDS: "Technology Assessment, Biomedical"[Mesh]; "Economics, Medical"[Mesh]; "EvidenceBased Medicine"[Mesh].

\section{REFERENCIAS BIBLIOGRÁFICAS}

Adam, P. \& Permanyer-Miralda, G. Research in health sciences, co-responsibility and social impact. Med. Clin. (Barc.), 141(6):254-6, 2013.

Banta, H. D.; Behney, C. J. \& Willems, J. S. Toward Rational Technology in Medicine: Considerations for Health Policy. New York, Springer, 1981

Castillo-Riquelme, M. \& Santelices, C. E. Foundations for the institutionalization of health technology assessment in Chile. Rev. Med. Chil., 142 Suppl. 1:S504, 2014.

Centre for Evidence-Based Medicine (CEBM). OCEBM Levels of Evidence. Oxford, Centre for Evidence-Based Medicine, 2009. Disponible en: http:// www.cebm.net/ocebm-levels-of-evidence

Cicchetti, A.; Berrino, A.; Casini, M.; Codella, P.; Facco, G.; Fiore, A.; Marano, G.; Marchetti, M.; Midolo, E.; Minacori, R.; Refolo, P.; Romano, F.; Ruggeri, M.; Sacchini, D.; Spagnolo, A. G.; Urbina, I.; Vaglio, S.; Grazzini, G. \& Liumbruno, G. M. Health Technology Assessment of pathogen reduction technologies applied to plasma for clinical use. Blood Transfus., 14(4):287386, 2016.

Conde Olasagasti, J. L. Evaluacion de Tecnologías Médicas Basada en la Evidencia. Madrid, Agencia de Evaluación de Tecnologías Sanitarias, Instituto de Salud Carlos III, Ministerio de Sanidad y Consumo, 1998. Disponible en: http://www.isciii.es/ISCIII/es/contenidos/fd-el-instituto/fd-organizacion/ fd-estructura-directiva/fd-subdireccion-general-evaluacion-fomentoinvestigacion/fd-centros-unidades/fd-agencia-evaluacion-tecnologias-sanitarias/fd-publicaciones-aets/evaluacion_tecnologias.pdf

Di Napoli, W. A.; Nollo, G.; Pace, N. \& Torri, E. Can clinical use of Social Media improve quality of care in mental Health? A Health Technology Assessment approach in an Italian mental health service. Psychiatr. Danub., 27 Suppl. 1:S103-10, 2015.

Espinoza, M. A. \& Cabieses, B. Equidad en salud y evaluación de tecnologías sanitarias en Chile. Rev. Med. Chile, 142 Suppl. 1:S45-9, 2014.

Foster, R. N. The S-Curve: A New Forecasting Tool. Londres, Macmillan, 1986.

Gagnon, M. P. Hospital-based health technology assessment: developments to date. Pharmacoeconomics, 32(9):819-24, 2014.

Gagnon, M. P.; Desmartis, M.; Poder, T. \& Witteman, W. Effects and repercussions of local/hospital-based health technology assessment (HTA): a systematic review. Syst. Rev., 3:129, 2014.

Goeree, R. \& Diaby, V. Introduction to health economics and decision-making: Is economics relevant for the frontline clinician? Best Pract. Res. Clin. Gastroenterol., 27(6):831-44, 2013.

Gray, E. \& Lorgelly, P. K. Health economics education in undergraduate medical degrees: an assessment of curricula content and student knowledge. Med. Teach., 32(5):392-9, 2010.

Guyatt, G.; Schunëmann, H.; Cook, D.; Jaeschke, R.; Pauker, S.; Bucher, H. \& American College of Chest Physicians. Grades of recommendation for antithrombotic agents. Chest, 119(1 Suppl.):3S-7S, 2001.

Health Quality Ontario. Fecal microbiota therapy for Clostridium difficile infection: A health technology assessment. Ont. Health Technol. Assess. Ser, 16(17):1-69, 2016a.

Health Quality Ontario. Percutaneous ventricular assist devices: A health technology assessment. Ont. Health Technol. Assess. Ser, 17(2):1-97, 2017.

Health Quality Ontario. Skin Testing for Allergic Rhinitis: A Health Technology Assessment. Ont. Health Technol. Assess. Ser., 16(10):1-45, 2016b.

Ju, H. \& Hewson, K. Health technology assessment and evidence-based policy making: Queensland Department of Health experience. Int. J. Technol. Assess. Health Care, 30(6):595-600, 2014.
Kuhn-Barrientos, L. Health Technology Assessment: conceptual framework and international perspective. Rev. Med. Chile, 142 Suppl. 1:S11-5, 2014.

Lysdahl, K. B.; Oortwijn, W.; van der Wilt, G. J.; Refolo, P.; Sacchini, D.; Mozygemba, K.; Gerhardus, A.; Brereton, L. \& Hofmann, B. Ethical analysis in HTA of complex health interventions. B. M. C. Med. Ethics, 17:16, 2016.

Manterola, C.; Pineda, V.; Vial, M. \& Grupo MInCir. Efectividad del tratamiento laparoscópico de la colelitiasis y la coledocolitiasis. Revisión global de la evidencia. Rev. Chil. Cir., 59(3):198-207, 2007a.

Manterola, C.; Pineda, V.; Vial, M. \& Grupo MInCir. Efectividad del tratamiento laparoscópico de quistes y tumores hepáticos. Revisión global de la evidencia. Rev. Chil. Cir., 59(4):264-71, 2007b.

Manterola, D. C.; Urrutia, S. \& Otzen, H. T. Health-related quality of life. Measurement tools to assessing upper gastrointestinal surgery outcomes. Int. J. Morphol., 31(4):1517-23, 2013.

Manterola, D. C.; Urrutia, V. S. \& Otzen, H. T. Health-related quality of life. Measurement tools to assessing upper gastrointestinal surgery outcomes. Rev. Chil. Cir., 66(3):274-82, 2014.

Oppong, R.; Mistry, H. \& Frew, E. Health economics education in undergraduate medical training: introducing the health economics education (HEe) website. B. M. C. Med.Educ., 13:126, 2013.

Organización Panamericana de la Salud (OPS). Organización Mundial de la Salud (OMS). Evaluación de Tecnologías Sanitarias (ETS). Washington D. C., Organización Panamericana de la Salud, 2017. Disponible en: http:// w w w. paho.org/h q/index.php? o ption $=\mathrm{com}$ content\&view $=$ article \&id=9229\%3 A2013-tecnologiassanitarias\&catid $=5870 \% 3$ Aassessment $\&$ Itemid $=41687 \&$ lang=es

Organización Panamericana de la Salud (OPS). Organización Mundial de la Salud. El desarrollo de la evaluación de las tecnologías en salud en América Latina y el Caribe. $4^{\mathrm{a}}$ ed. Washington D. C., Organización Panamericana de la Salud, 2000. Disponible en: http://apps.who.int/medicinedocs/ documents/s16572s/s16572s.pdf

Rycroft, C. E.; Hirst, M.; Dunlop, W. C.; Pirk, O.; Mullins, D. \& Akehurst, R. The Suitability of end point designs for health technology assessment in chronic pain studies. Value Health, 18(8):987-93, 2015.

Scottish Intercollegiate Guidelines Network. SIGN 50: A Guidelines Developers' Handbook. Edinburgh, SIGN, 2004.

Tanvejsilp, P. \& Ngorsuraches, S. Defining the scope of health technology assessment and types of health economic evaluation. J. Med. Assoc. Thai., 97 Suppl. 5:S10-6, 2014.

Teutsch, S. \& Berger, M. L. Evidence synthesis and evidence-based decision making: related but distinct processes. Med. Decis. Making, 25(5):487-9, 2005.

Vallejos, C.; Bustos, L.; de la Puente, C.; Reveco, R.; Velásquez, M. \& Zaror, C. The main methodological aspects in Health Technology Assessment. Rev. Med. Chil., 142 Suppl. 1:S16-21, 2014.

Yglesias Bedoya, A. Análisis Comparativo de Políticas y Normatividad Relacionada con la Evaluación de Tecnología Sanitaria en los Países de la Sub Región Andina. Lima, Organismo Andino de Salud-Convenio Hipólito Unanue, 2007. Disponible en: http://www.orasconhu.org/sites/default/files/ AnalisisTecnolog\%20Sanitaria.pdf

Youngkong, S. Application of HTA research on policy decision-making. J. Med. Assoc. Thai., 97 Suppl. 5:S119-26, 2014.

Dirección para correspondencia:

Dr. Carlos Manterola Delgado

Departamento de Cirugía y CEMyQ.

Universidad de La Frontera.

Manuel Montt 112, oficina 408

Temuco

CHILE

E-mail: carlos.manterola@ufrontera.cl

Recibido : 28-06-2017

Aceptado: 30-01-2018 